\title{
Virtual Prototyping Technique in Active Learning
}

\author{
Mariana FRATU \\ Transilvania University of Brasov, Romania, mariana.fratu@unitbv.ro \\ Iulian Dorin SBANCA \\ Transilvania University of Brasov, Romania, dorinel.sbanca@unitbv.ro
}

\begin{abstract}
In this paper, we continue to develop the virtual prototypes models and their testing for active learning support with Erasmus students. The personalized applications selected by a tutor are used directly for performing active learning at the university's detached workplace. When using the virtual prototypes the main strategy of the informatics support is based on the developing informatics tools which are adapted for a pedagogical - engineering content. Online learning outcomes materials, as virtual prototypes, were created last years and tested on the informatics environment. Some of them were also investigated as the "active elements" for active learning, especially for writing stage rapports including practical projects. The practice showed that the testing of issues such as the practical placement stage work can result in an active learning process. The domain of investigation is the virtual prototyping. The virtual product development replaces traditional material object test with digitized form product by building product's digital model. It offers to analyze product's static and dynamic characteristics on the circumstance of digital state, and rebuild and improve the original design. Virtual prototype technology is a kind of engineering method which can shorten new product development cycles by replacing cost physical prototype with virtual digital model. Virtual prototyping technique, as method for active learning informatics support, requires the further refinement and also the enhancement of students' informatics skills, as well.
\end{abstract}

\section{Keywords}

active learning, virtual prototypes, practical projects

\section{Introduction}

The paper deals with a method used many years for example based learning for the virtual prototyping. One of the starting point and subject was the Erasmus exchanges in the European Union. The faithful collaboration Transilvania University of Brasov in Romania walks with Polytechnic University of Hauts-de-France, previously known as University of Valenciennes and Hainaut-Cambrésis since 20 years. The relationships with foreign universities have been increased since that date. It means four French students a year in the bachelor of technology course got a three months placement in Romania supervised by the authors.

The investigation domain is the virtual prototyping. They are applying this learning method described in this paper. Virtual prototype technique is a kind of engineering method which can shorten new product development cycles by replacing cost physical prototype with virtual digital model. The subject detailed here deals with a virtual prototyping using Delphi programming environment. The example based active learning method is thus applied.

There are several potential advantages of active based learning programs, including the ability for people to learn from their homes and study without the assistance of an instructor. As the use of computers in life is increasing, all learning methods and strategies should be renewed to match with the advancement of technology. It means that videos, texts, lessons, answered homeworks in electronics format should be easily downloaded by any new learners. Active learning requires students to do meaningful learning activities and think about what they are doing.

The method proposed takes three main steps. The first step offers the connected student to download as many examples as he wants. Any example is given in an executable format only. The student launches the example, takes notes and comments on the scenario and writes down new ideas on a working platform.

The second step is devoted to the discovery of the application language. A given example is given to the student including the source code. At that moment, the student can slightly change any code line and check at once the positive or negative effects. 
In the third step the student uses his own imagination in order to create a new product and sometimes to obtain a patent. The most popular way to encourage innovation is to offer students recognition for innovative ideas by allocating time for innovative work and by satisfying with opportunities for career progression. That is to promote innovation is the biggest challenge in the use of virtual prototyping technique. That means having the ability to demonstrate tangible business benefits of new automatic systems. It may be necessary for students - expectations engineers - to win theirs confidence on virtual prototype first, before moving on to real/physical system- adopting virtual prototyping learning method brings student focused on to master new systems. During the stage the students are preparing to work in the innovative companies which are known for encouraging innovation by freeing up its staff to spend one day a week developing their own innovative projects.

\section{Methodology}

The virtual prototyping technique -VPT in short- can help students/graduates develop knowledge and skills to pursue such as software applications developer job or software applications engineer job. The virtual product development replaces traditional material object test with digitized form product by building product's digital model. The virtual prototyping technique - based active learning is a term that describes the way to use computers as a central instrument involving the user imagination. This teaching approach takes advantage of the interactive elements of computer software, along with the computer's ability to present many different kinds of applications.

The impact of computers on our lives encourages the development of new software. The VPT is a kind of engineering method which can shorten new product development cycles by replacing cost physical prototype with virtual digital model.

The authors are running for decades to the vanishing motivation of their students. Since 20 years, they consumed their energy to find new learning methods. Active learning based on examples has been used as a tool in the university laboratories of a more traditional team experience. It has also been used as an elementary learning method, especially in many online educational programs.

The use of computers for learning has generally increased with the advancement of technology. The ability of computer hardware to process and present many different media types has allowed for more complex computer learning strategies [1, 2,3].

An example would be a training module that uses virtual prototype as model. Code source and pictures are often handled in an interactive manner, and sometimes they can almost be like games, depending on the style of the implementation.

A very high-quality education experience can potentially be implemented in a computer-based virtual environment and then distributed widely around the world to people of all economic backgrounds. With computers, the students can potentially spend as much time as necessary to understand a given subject before moving on. Virtual prototypes examples based active learning allows students to have the personal initiative they need. Virtual prototyping technique based active learning often brings up the fact that it is relatively common for a program to mix virtual prototypes examples to obtain a new application [4].

This learning strategy requires students to revisit multiple virtual prototypes created before by former students for a stronger understanding of that they must do. Working through these examples and discussing with a partner, helps students to gain a deeper understanding of the subject.

The students may work together in pairs or small groups to discuss and compare their ideas. But the students can be also asked to work individually. The reason is such that they can prepare their virtual prototype based on personal ideas. Each student will be able to create its personalized application that turns him proud of its work.

With virtual prototyping, the development of a product moves directly from design to testing and analysis, and then finally to build [5]. The VPT allows for the digital exploration the carries out any product optimization and complete behavioral simulation before it is in reality built, decreasing the number of rebuilds necessary and minimizing material waste.

The VPT can be used not only to observe and examine products before fabrication, but also to test products and parts before physical product build, to test systems and infrastructure strategy before implementation. 


\section{Results}

In the virtual environment, in order to view the projected images, the 3-D scene projections are created using specialized software. These are known for their advanced visualization solutions for 3-D applications designed to display virtual prototypes. It offers to analyze product's static and dynamic characteristics on the circumstance of digital state, and rebuild and improve the original design. The virtual prototype technology carries out any product optimization.

\subsection{Virtual Prototype of the Experimental Production Line}

In the case of 3-D virtual objects such as a manufacturing line, designers can observe around the 3-D objects exactly as they would in the real world (Fig. 1).

The paper ends with two examples of virtual prototypes created in order to support technical design and draws some conclusions.

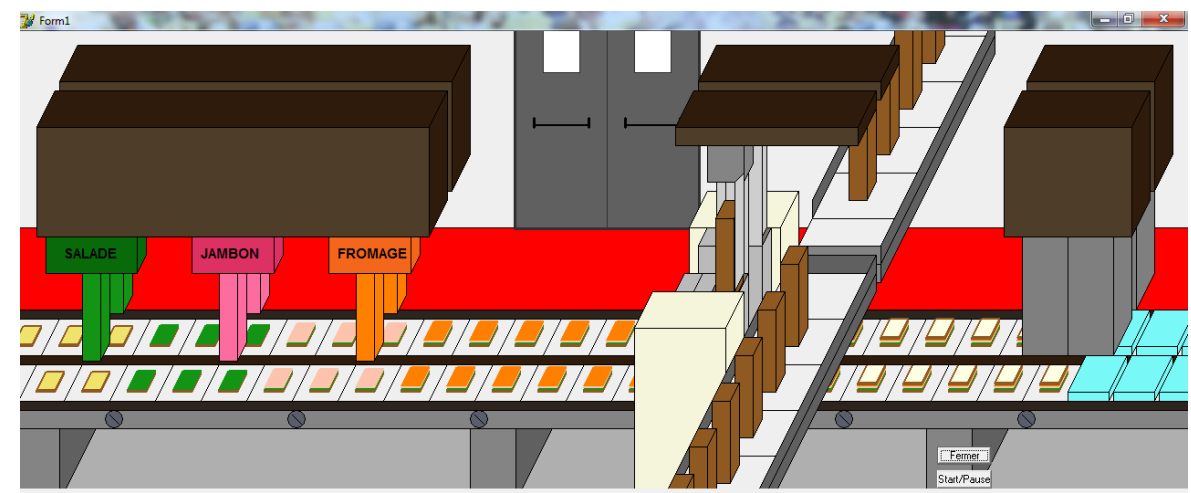

Fig. 1. Virtual model of a manufacturing line

\subsection{Virtual prototype of the experimental}

The designer can essentially experience a 3-D environment while interact with the virtual world accordingly. Product or infrastructure testing and analysis become far more effective when the viewer can interact with the 3-D world in real time; such is the case of haptic interfaces, another viable example of the VPT. Haptic technology allows the collaborative work between virtual world and physical world (Fig. 2).

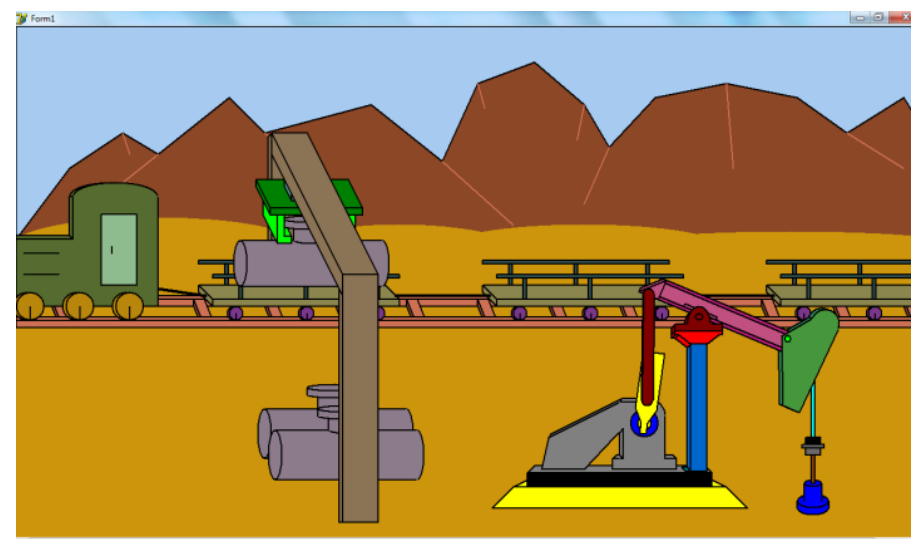

Fig. 2. A virtual prototype of the experimental installation to exploring of an area for natural resources

A virtual prototype allows product design teams to view moving parts within a system, ensure that elements operate together impeccably, and validate real world performance; all before manufacturing always takes place. The VPT brings virtual prototypes to a whole new level by permitting the designer to experience an entire manufacturing process, complete with interactive features and real time tracking of the components' movements. The VPT in all forms allow a company to cut down on costs, minimize rebuilds, and move products to market more efficiently. 


\section{Conclusion}

Active learning is generally defined as any instructional method that engages students in the learning process. With the development of the virtual technologies students can explore more realistic 3D environments and interact with artificial systems of the environment.

To end their bachelor of technology course, the French students in electrical engineering domain follow a 3 months placement at the Romanian university. They are supervised by the teachers mentioned in the paper head. Applied active learning a project should engage students in solving semistructured problems, demand skills expected in high-performance work organizations, require students to develop organisational and self-management skills.

The interaction allowed by virtual prototypes models could bring an end to passive learner attitudes which are often found in traditional academic teaching situations. In addition, virtual prototypes technology could be applied as a complement to traditional academic teaching, leading to better understanding of the process behavior, whether in training, in education or in professional practice.

The virtual prototypes can be proactive about building a work environment that's ready for the future. Thus, active learning - based virtual prototypes technique - may well induce students to consider this knowledge as elementals in their future professional activity, while to establishing the link between virtual technologies and engineering theory.

Virtual environments offer new opportunities and possibilities for engineers. The virtual product development replaces traditional material object test with digitized form product by building product's digital model. It offers to analyze product's static and dynamic characteristics on the circumstance of digital state, and rebuild and improve the original design.

The innovative design technologies would need to ensure that the end users achieve the utilization of its abilities in optimization of the projects.

The applications with these characteristics make the advantage of using visual programming and virtual prototyping technique as tool of active learning process. There are several potential advantages of examples-based active learning programs, including the ability for people to learn from their homes and study without the assistance of an instructor.

The software applications development can be used to help students/designers to develop and implement the virtual prototypes in engineering. Typical product development cycles can be expensive procedures, particularly when physical prototypes are required. When a physical model is involved, the product must be designed, built and tested.

Physical prototypes must be built and re-built after every unsuccessful test, making them a less than optimal choice for a company that values efficiency.

As future work we will apply the approach proposed in this paper to other buildings, with the aim of demonstrating its applicability in different contexts.

\section{References}

1. Cai J.-J., et al. (2019): Multi-View Active Learning for Video Recommendation. In Proceedings of IJCAI-19, Macao, China, https://www.ijcai.org/proceedings/2019/0284.pdf

2. Lee J., et al. (2018): Collaborative deep metric learning for video understanding. In KDD '18: The 24th ACM SIGKDD International Conference on Knowledge Discovery \& Data Mining, ACM ISBN 978-1-4503-5552-0/18/08, https://doi.org/10.1145/3219819.3219856, http://www.joonseok.net/papers/cdml.pdf

3. Chen X., et al. (2018): Temporal hierarchical attention at category-and item-level for micro-video click-through prediction. In MM '18 Proceedings of the 26th ACM international conference on Multimedia, ISBN 978-1-45035665-7, p. 1146-1153, Seoul, Republic of Korea, https://doi.org/10.1145/3240508.3240617

4. Eison J. (2010): Using Active Learning Instructional Strategies to Create Excitement and Enhance Learning. http://citeseerx.ist.psu.edu/viewdoc/download?doi=10.1.1.456.7986\&rep=rep1\&type=pdf

5. Bécar J.-P., et al. (2015): Three Experiments of Learning by Examples. In Proceedings of INTED2015 Conference, ISBN 978-84-606-5763-7, p. 1293-1302, Madrid, Spain, https://www.academia.edu/21842531/ THREE EXPERIMENTS OF LEARNING BY EXAMPLES 